\title{
Stereotactic body radiation therapy versus multi-fraction radiation therapy for bone metastases
}

\author{
Charles B. Simone II \\ New York Proton Center, New York, NY, USA \\ Correspondence to: Charles B. Simone II, MD. New York Proton Center, 225 East 126th Street, New York, NY 10035, USA. \\ Email: csimone@nyproton.com. \\ Comment on: Nguyen QN, Chun SG, Chow E, et al. Single-Fraction Stereotactic vs Conventional Multifraction Radiotherapy for Pain Relief in \\ Patients With Predominantly Nonspine Bone Metastases: A Randomized Phase 2 Trial. JAMA Oncol 2019;5:872-8.
}

Submitted Jul 08, 2019. Accepted for publication Jul 11, 2019.

doi: 10.21037/apm.2019.07.06

View this article at: http://dx.doi.org/10.21037/apm.2019.07.06

Bone metastases remain one of the most common sites of metastatic cancer spread. Bone metastases can cause considerable pain for patients, which can lead to anxiety, a sense of hopelessness, and depression $(1,2)$. While adequate pain control from cancer-related pain can often be achieved through the use of individualized pain management strategies optimizing analgesic medications (3), many patients require palliative radiotherapy as a means to most optimally control their pain, prevent bone fraction or spinal cord compression, or provide durable tumor control. While the recently evolving landscape of systemic therapies has changed the treatment paradigm for many metastatic patients, the improved response rates and even prolonged survivals seen with target therapies and immunotherapies provide further justification for aggressive pain management of painful bone metastasis and for a local therapy option that provides durable tumor control in metastatic patients who are achieving longer overall survivals.

The optimal dose-fractionation regimen for palliative radiotherapy for bone metastasis remains controversial. In the past half century, numerous randomized trials in palliative oncology have assessed outcomes with various radiation dose-fractionation schemes. These trials have been jointly analyzed in multiple systematic reviews and meta-analyses that have concluded that single-fraction radiotherapy ( $8 \mathrm{~Gy} \times 1$ fraction) is equivalent in pain relief to longer radiotherapy courses (such as $4 \mathrm{~Gy} \times$ 5 fractions, 4 Gy $\times 6$ fractions, 3 Gy $\times 10$ fractions, or even more prolonged fractionation schemes) for uncomplicated bone metastases, which includes those without pathologic fracture, spinal cord compression, neuropathic pain, or soft tissue component (4-7). When delivering palliative radiotherapy using single- or multi-fraction regimens for uncomplicated bone metastases, approximately $25-40 \%$ of patients achieve complete pain relief, and the overall pain response rate is approximately $75-90 \%(4-8)$.

Complete and overall response rates, overall side effect profiles, and rates of subsequent spinal cord compression across studies generally are not significantly difference between single- and multi-fraction radiotherapy, whereas some analyses have demonstrated higher rates of pathological fractures after single-fraction radiotherapy, and the rate of retreatment is generally higher for singlefraction radiotherapy (4-7). These higher retreatment rates are perhaps due to less durable pain control and also radiation oncologists' increased willingness and comfort with retreatment after relatively low dose, single-fraction radiotherapy (8). Notably, even with accounting for higher retreatment rates, single-fraction radiotherapy has been demonstrated to be more cost-effective than multi-fraction radiotherapy for uncomplicated bone metastases (9). With conventional radiotherapy, therefore, there is a tradeoff between multi-fraction regimens that deliver higher total radiotherapy doses that potentially have more durable pain control versus single-fraction regimens are more convenient for patients and their caregivers, less resource intensive on radiation oncology clinics, more cost effective, and better able to be integrated before or between cycles of systemic therapy.

Stereotactic body radiation therapy (SBRT) may allow 
for the best of both approaches-a short and convenient treatment course that delivers high-dose radiotherapy that can provide durable treatment responses. SBRT is an advanced radiotherapy modality that has emerged as an option for treating patients with symptomatic bone metastases (10). SBRT delivers an ultrahigh dose of radiation per treatment fraction to a defined target. SBRT imparts ablative radiotherapy doses and often higher biologic-equivalent doses (BED) of radiotherapy than are achievable with conventionally fractionated radiation, which often translates into superior tumor killing compared with conventional radiotherapy. SBRT also achieves a rapid dose falloff beyond the tumor, allowing for a high degree of normal tissue sparing. Reproducible target localization is critical when delivering SBRT, and the use of fiducial markers or image-guided radiotherapy to verify correct patient positioning and tumor localization before each treatment should be employed, methods that are often unnecessary when delivering conventionally fractionated palliative radiotherapy (11).

As the majority of patients will experience few side effects and receive significant clinical benefit from conventional palliative radiotherapy with commonly delivered regimens such as 8 Gy $\times 1$ fraction, 4-5 Gy $\times 5-6$ fractions, and 3 Gy $\times 10$ fractions, determining which patients could have an appreciable benefit from SBRT currently is not defined. SBRT in patients with metastatic disease can be particularly beneficial in the reirradiation setting for which disease or symptoms have recurred following an initial course of palliative radiotherapy, as SBRT can limit retreatment doses to normal tissues and thus may limit side effects while still achieving palliative relief (12). SBRT is also particularly suited for advanced stage patients with oligoprogressive or oligometastatic disease. In this setting, more than palliating symptoms, SBRT can achieve durable tumor control and can improve progression-free survival and even overall survival compared with systemic therapy alone (13-15).

The benefit of SBRT cover conventional radiotherapy for patients with symptomatic bone metastases is currently an area of active investigation. RTOG 0631, a randomized phase II-III trial of uncomplicated spinal bone metastases without cord compression, is assessing complete pain relief following single-fraction SBRT to 16-18 Gy versus singlefraction conventional radiotherapy to $8 \mathrm{~Gy}(16)$. This trial has completed accrual, and when it is reported it could change the treatment approach for palliative patients. Until now, however, no randomized trial comparing SBRT to multi-fractionated radiotherapy for patients with painful bone metastases has been completed to inform clinicals of the true utility and benefits of SBRT.

Investigators from University of Texas MD Anderson Cancer Center conducted a randomized phase II noninferiority trial where they enrolled 160 adult patients with painful bone metastasis from $09 / 2014$ to $06 / 2018$ (17). The study focused on uncomplicated bone metastases, as patients with spinal cord compression and pathologic fractures were excluded. Patients were treated with SBRT in a single fraction (12 Gy for $\geq 4 \mathrm{~cm}$ lesions or $16 \mathrm{~Gy}$ to $<4 \mathrm{~cm}$ lesions) or with multi-fraction conventional radiotherapy (30 Gy in 10 fractions). The primary endpoint of the study was pain response, as defined by a combination of pain score and analgesic usage.

Among evaluable patients receiving treatment per protocol (107 of 160 enrolled patients), more patients in the SBRT arm were classified as pain responders (complete or partial responses) at 2 weeks ( $62 \%$ vs. $36 \%, \mathrm{P}=0.01)$, 3 months ( $72 \%$ vs. $49 \%, \mathrm{P}=0.03)$, and 9 months $(77 \%$ vs. $46 \%, \mathrm{P}=0.03)$, whereas response rates were similar at 1 month $(68 \%$ vs. $45 \%, \mathrm{P}=0.21)$ and 6 months $(68 \%$ vs. $61 \%, \mathrm{P}=0.78)$. Intention-to-treat analyses also showed similar to higher response rates for SBRT at 2 weeks $(\mathrm{P}=0.03), 1$ month $(\mathrm{P}=0.18), 3$ months $(\mathrm{P}=0.05), 6$ months $(\mathrm{P}=0.89)$, and 9 months $(\mathrm{P}=0.06)$. Furthermore, local control rates at both 1 and 2 years were higher among patients in the SBRT arm, and the cumulative incidence of local failures at 24 months were $0 \%$ vs. $10 \%(\mathrm{P}=0.02)$. This uniform local control with SBRT is in keeping with prior analyses showing excellent rates of durable control with SBRT for bone metastases $(10,18)$. Reirradiation rates also trended lower with SBRT (1-year: 0\% vs. 3\%; 2-year: 0\% vs. $5 \% ; \mathrm{P}=0.10)$. Progression-free survival rates were higher with SBRT (1-year: $100 \%$ vs. $90.5 \%$; 2 -year: $100 \%$ vs. $75.6 \% ; \mathrm{P}=0.01)$, whereas the overall survival was identical between arms (17).

These benefits of SBRT were not at the expense of toxicity, as there were no differences in treatment-related adverse events or quality-of-life scores between arms. In fact, both treatment arms led to similarly improved qualityof-life scores. Interestingly, pain response rates among SBRT patients were higher with 16 Gy than with 12 Gy at 3 months (62\% vs. $30 \%$ ), and higher-dose SBRT also provided the most durable response (9-month response rates were $43 \%$ vs. 13\%) (17). While this is in keeping with the known finding that higher BED of SBRT correlate with higher tumor response rates and improved outcomes $(19,20)$, the results in this study may also be confounded by higher 
doses in the trial being delivered to smaller lesions that would have been more likely to respond to treatment.

The authors should be commended for completing the first-ever randomized trial of SBRT in this patient population. While several limitations of this trial exist, including several patients not receiving treatment per randomization, considerable patient attrition during the course of the trial, and this being a single-center study, this trial further establishes high-dose, single-fraction SBRT as a standard treatment option that is safe and effective for patients with painful bone metastases. The authors demonstrated that higher BEDs delivered with SBRT can achieve improved short- and long-term pain responses, and that SBRT might be the optimal approach for select patients with excellent performance statuses, longer life expectancies, and limited bone metastases. While providers should consider patient prognosis when determining which radiotherapy dose-fractionation scheme to deliver, this underlies the challenge of predicting survival for patients with metastatic disease, for which physicians often significantly overestimate life expectancy (21).

Providers treating patients with advanced malignancies and bone metastases causing pain should consider early referral to palliative care services to improve clinical outcomes and patient quality of life (22) and to radiation oncologists for evaluation of palliative radiotherapy to improve symptom scores, including pain. With the rise in single-fraction radiotherapy regimens, either delivered as conventional treatment or as SBRT, radiation therapy can now more easily (and potentially more synergistically) than ever be integrated into a patient's overall treatment course with systemic therapy (23) and can achieve a speedy pain response when delivered for painful uncomplicated bone metastases that can improve quality of life for patients (24). Multi-center randomized trials are encouraged and could establish SBRT as the preferred treatment approach for select patients with painful, uncomplicated bone metastases.

\section{Acknowledgments}

None.

\section{Footnote}

Conflicts of Interest: The author has no conflicts of interest to declare.

Ethical Statement: The author is accountable for all aspects of the work in ensuring that questions related to the accuracy or integrity of any part of the work are appropriately investigated and resolved.

\section{References}

1. Spiegel D, Sands S, Koopman C. Pain and depression in patients with cancer. Cancer 1994;74:2570-8.

2. Simone CB 2nd, Jones JA. Palliative care for patients with locally advanced and metastatic non-small cell lung cancer. Ann Palliat Med 2013;2:178-88.

3. Simone CB 2nd, Vapiwala N, Hampshire MK, et al. Cancer patient attitudes toward analgesic usage and pain intervention. Clin J Pain 2012;28:157-62.

4. Chow E, Zeng L, Salvo N, et al. Update on the systematic review of palliative radiotherapy trials for bone metastases. Clin Oncol (R Coll Radiol) 2012;24:112-24.

5. Wu JS, Wong R, Johnston M, et al. Meta-analysis of dose-fractionation radiotherapy trials for the palliation of painful bone metastases. Int J Radiat Oncol Biol Phys 2003;55:594-605.

6. Sze WM, Shelley M, Held I, et al. Palliation of metastatic bone pain: single fraction versus multifraction radiotherapy - a systematic review of the randomised trials. Cochrane Database Syst Rev 2004;(2):CD004721.

7. Chow E, Harris K, Fan G, et al. Palliative radiotherapy trials for bone metastases: a systematic review. J Clin Oncol 2007;25:1423-36.

8. Jones JA, Simone CB 2nd. Palliative radiotherapy for advanced malignancies in a changing oncologic landscape: guiding principles and practice implementation. Ann Palliat Med 2014;3:192-202.

9. Konski A, James J, Hartsell W, et al. Economic analysis of radiation therapy oncology group 97-14: multiple versus single fraction radiation treatment of patients with bone metastases. Am J Clin Oncol 2009;32:423-8.

10. Bedard G, McDonald R, Poon I, et al. Stereotactic body radiation therapy for non-spine bone metastases--a review of the literature. Ann Palliat Med 2016;5:58-66.

11. Simone CB 2nd, Wildt B, Haas AR, et al. Stereotactic body radiation therapy for lung cancer. Chest 2013;143:1784-90.

12. Mantel F, Flentje M, Guckenberger M. Stereotactic body radiation therapy in the re-irradiation situation--a review. Radiat Oncol 2013;8:7.

13. Gomez DR, Tang C, Zhang J, et al. Local Consolidative Therapy Vs. Maintenance Therapy or Observation for Patients With Oligometastatic Non-Small-Cell Lung 
Cancer: Long-Term Results of a Multi-Institutional, Phase II, Randomized Study. J Clin Oncol 2019;37:1558-65.

14. Palma DA, Olson R, Harrow S, et al. Stereotactic ablative radiotherapy versus standard of care palliative treatment in patients with oligometastatic cancers (SABRCOMET): a randomised, phase 2, open-label trial. Lancet 2019;393:2051-8.

15. Iyengar $\mathrm{P}$, Wardak Z, Gerber DE, et al. Consolidative Radiotherapy for Limited Metastatic Non-Small-Cell Lung Cancer: A Phase 2 Randomized Clinical Trial. JAMA Oncol 2018;4:e173501.

16. Ryu S, Pugh SL, Gerszten PC, et al. RTOG 0631 phase $2 / 3$ study of image guided stereotactic radiosurgery for localized (1-3) spine metastases: phase 2 results. Pract Radiat Oncol 2014;4:76-81.

17. Nguyen QN, Chun SG, Chow E, et al. Single-Fraction Stereotactic vs Conventional Multifraction Radiotherapy for Pain Relief in Patients With Predominantly Nonspine Bone Metastases: A Randomized Phase 2 Trial. JAMA Oncol 2019. [Epub ahead of print].

18. Erler D, Brotherston D, Sahgal A, et al. Local control and fracture risk following stereotactic body radiation therapy for non-spine bone metastases. Radiother Oncol

Cite this article as: Simone CB 2nd. Stereotactic body radiation therapy versus multi-fraction radiation therapy for bone metastases. Ann Palliat Med 2019;8(4):360-363. doi: 10.21037/apm.2019.07.06
2018;127:304-9.

19. Onishi H, Shirato H, Nagata Y, et al. Hypofractionated stereotactic radiotherapy (HypoFXSRT) for stage I nonsmall cell lung cancer: updated results of 257 patients in a Japanese multi-institutional study. J Thorac Oncol 2007;2:S94-100.

20. Choi JI, Simone CB 2nd. Stereotactic body radiation therapy versus surgery for early stage non-small cell lung cancer: clearing a path through an evolving treatment landscape. J Thorac Dis 2019;11:S1360-5.

21. Christakis NA, Lamont EB. Extent and determinants of error in doctors' prognoses in terminally ill patients: prospective cohort study. BMJ 2000;320:469-72.

22. Gaertner J, Lutz S, Chow E. Early palliative care: philosophy vs. reality. Ann Palliat Med 2015;4:87-8.

23. Simone CB 2nd, Burri SH, Heinzerling JH. Novel radiotherapy approaches for lung cancer: combining radiation therapy with targeted and immunotherapies. Transl Lung Cancer Res 2015;4:545-52.

24. Malek L, Chow E, Simone CB 2nd. Speedy response can be achieved from palliative radiotherapy in the treatment of painful uncomplicated bone metastases. Ann Palliat Med 2017;6:S120-1. 